happened after the United Kingdom's thermonuclear explosive capabilities had been demonstrated, and coincided with the decision of the president of the United States to attempt to restart military nuclear collaboration. It also led to the exchange of a can-do culture within the programme for a strengthened safety one. In view of the Chernobyl experience, Britain may count itself fortunate that this happened before its civil nuclear power reactors were commissioned, and with such minimal environmental and medical consequences.

John Simpson is at the Mountbatten Centre for International Studies, Department of Politics, University of Southampton, Southampton SO9 $5 \mathrm{NH}$, UK.

\section{Filling in the}

\section{gaps}

\section{David N. Schramm}

The Hidden Universe. By Roger J. Tayler. Ellis Horwood: 1991. Pp. 213. £30 (hbk), $£ 15.99$ (pbk).

Cosmology has become one of the most active and exciting topics in modern physical science. Never before have so many experiments and observations had possible cosmological implications. Although modern cosmology probably started in 1929 with Edwin Hubble's observations of an expanding Universe, the next important observation of immense cosmological impact was not until the discovery by Arno Penzias and Robert Wilson in 1965 of the microwave background radiation. In the past decade the pace of discovery has quickened enormously, with accelerator experiments and telescopes on the ground, as well as satellites in space, giving us information that has cosmological relevance. This has enabled cosmological studies to move from the more philosophical side of intellectual endeavour to become a genuine physical science where hypotheses are checked by making predictions that lead to actual measurements.

Although my personal interest no doubt biases my view, I feel it is safe to say that, along with the microwave background, the light elements have had the most lasting recent effects. The cosmological nucleosynthesis predictions for the abundances of the light elements, ${ }^{1} \mathrm{H},{ }^{2} \mathrm{H},{ }^{3} \mathrm{He},{ }^{4} \mathrm{He}$ and ${ }^{7} \mathrm{Li}$, span a range of almost 10 orders of magnitude and each is now observed, in primitive material, to be at its predicted value. Furthermore, it was shown that the amount of ${ }^{4} \mathrm{He}$ is related to the number of types of fundamental parti- cles. In particular, we now know that there could be no more than three families of neutrinos without exceeding the observed primordial abundance of helium. This number of neutrino families was directly observed last year at the LEP collider at the European Laboratory for Particle Physics (CERN) in Geneva, Switzerland. So cosmology is now making predictions that can be checked with particle accelerators as well as telescopes.

Roger Tayler was one of the pioneers in cosmological nucleosynthesis calculations. Even before the microwave background was first measured, Tayler's work with Fred Hoyle showed that primordial nucleosynthesis would convert an appreciable fraction of the mass of the Universe into ${ }^{4} \mathrm{He}$. Showing foresight, they even noted at the time a connection between the helium yield and neutrinos, although they did not explicitly calculate anything about this relationship. Clearly, Tayler has been one of the key players in the recent developments in cosmology.

Although there have been many books written over the past few years that have tried to convey the current level of excitement in the field to the nonscientifically educated public, little has been written for the more mathematically literate nonspecialist. The Hidden Universe nicely fills this need. Like several other recent books, it is an excellent and very readable presentation of the main topics in modern cosmology. But it differs from the others in that it is not afraid to use algebra. This, unfortunately, distances the book from the general public, but it makes it much more useful for scientists in other fields who wish to gain an insight into the excitement of cosmology today. Furthermore, the book is not meant as an advanced text or monograph for graduate students. Instead, it is written at a level that undergraduates or even advanced secondary school students can understand as long as they are familiar with elementary algebra.

Tayler presents the basic development of the current cosmological picture, leading his readers naturally into the central problems in current cosmology - namely, what constitutes the so-called dark matter in the Universe and how does large-scale structure, such as galaxies and 'great walls', arise from the apparently 'smooth' early Universe.

The need for some sort of exotic dark matter is a straightforward consequence of the light-element story. The lightelement abundances all give the remarkable fit mentioned above, but only if the density of ordinary neutron- and protonbased matter is relatively low. But other arguments imply that the overall density of the Universe is much higher, hence the need for some new exotic stuff. Tayler's arguments are not watered down, but are fully presented with the appropriate algebraic equations so that they can be followed.

A disappointment with the book is its lack of references. Although there is a good list of annotated suggestions for further reading in the back pages, there is no mention of where to go for further details on specific points. This drawback could limit the book's usefulness as a text in university courses. Tayler does in fact admit in the introduction that there will be very few citations of scientific contributions, but this sparseness is very noticeable when figures, taken from other published sources, are not fully cited. By contrast, the appendices, on such topics as degenerate matter, the virial theorem and thermodynamic equilibrium, present short, simple derivations of useful physics that are ideal for undergraduates or for scientists from other fields who need to refresh their memories. In short, The Hidden Universe provides a very readable and much needed introduction to modern cosmology for any algebraically literate reader. $\square$

David N. Schramm is in the Department of Physical Sciences, University of Chicago, Chicago, Illinois 60637, USA.

\section{Chemical controversies}

\section{W. P. Griffith}

Oxygen Chemistry. By Donald T. Sawyer. Oxford University Press: 1992. Pp. 223. $£ 30, \$ 35$.

THIS interesting and stimulating book represents very much an individual view. Even the first sentence in the preface could be taken by some chemists as controversial: "The book results from a conviction that oxygen is the most important element in the realm of chemistry". As R. J. P. Williams says in a perceptive introduction, "The author probably knows that he is being provocative [but he] is writing from deep knowledge and experience".

Sawyer rightly emphasizes the central role of oxygen in chemistry and provides a good overview of the principal oxygencontaining species (dioxygen, ozone, hydrogen peroxide, alkyl hydroperoxides, peracids; the hydroxyl, perhydroxyl, hydroperoxo, ozonide, oxo and superoxo anions, oxyanions; and also oxygen-containing radicals). The focus, appropriately, is on the reactivity of these species and on the kinetic and thermodynamic data that have been collected for them. In a rather curious and 